\title{
COGNIÇÃO E SOCIEDADE: UM OLHAR SOB A ÓPTICA DA LINGUÍSTICA COGNITIVA
}

\author{
Solange Coelho Vereza* \\ Universidade Federal Fluminense (UFF) \\ Instituto de Letras \\ Departamento de Letras Estrangeiras Modernas \\ Niterói, RJ, Brasil
}

\begin{abstract}
Resumo: Este trabalho propõe uma reflexão em torno da relação entre cognição e sociedade, sob a perspectiva da Linguística Cognitiva (LC). O conceito de cognição corporificada (embodied cognition), um dos pilares da LC, põe em xeque qualquer possibilidade de dicotomização entre os dois conceitos, uma vez que representações cognitivas como frames, Modelos Cognitivos Idealizados (MCIs) e metáforas conceituais seriam ancoradas na conceitualização de experiências tanto sensório-motoras, quanto socioculturais. A busca da compreensão da articulação entre o social e o cognitivo, entretanto, deve, segundo Gibbs (2001), sair do âmbito exclusivo do pensamento e encontrar o mundo. Por esta razão, propõe-se uma abordagem alinhada à recente tendência cognitivodiscursiva, que busca instrumentos teórico-metodológicos para sistematizar o estudo de tal articulação. Entre esses, a distinção entre sistema e uso, cognição episódica e estável, frames online e offline, e metáforas situadas e conceituais tem se mostrado teórica e analiticamente produtiva.
\end{abstract}

Palavras-chave: Sociocognição. Metáfora. Discurso.

Ao ser convidada para fazer parte de uma mesa-redonda intitulada Cognição $e$ Sociedade, no contexto da VII Conferência Linguagem e Cognição, que aconteceu na Universidade Federal da Paraíba, em agosto de 2015, busquei, entre os estudos que desenvolvia, ou entre os que pretendia desenvolver, aquele que mais poderia contribuir para o tema proposto. Os meus trabalhos específicos mais recentes (VEREZA, 2013a, 2013b, 2016) têm um norte em comum: a investigação da dimensão cognitivo-discursiva da metáfora em uso e dos frames nos quais ela se ancora (no caso de frames off-line) e ao mesmo tempo, cria (frames online) na linguagem em uso. Nesse sentido, como a relação, ou mesmo a interdeterminação, entre discurso e sociedade já é hoje quase um pressuposto consensual nos estudos contemporâneos da linguagem, sendo que o próprio conceito de discurso implica o uso socioculturalmente inserido do que era comumente conceituado como um sistema linguístico idealizado e, portanto, descontextualizado (sendo a langue saussuriana o protótipo desse conceito), um estudo sobre a metáfora no discurso pareceume atender o critério de coerência temática com a mesa-redonda proposta.

\footnotetext{
*Doutora em Linguística Aplicada e Estudos da Linguagem. E-mail: svereza@uol.com.br.
} 
No entanto, um dos fundamentos da Linguística Cognitiva (LC) que, inclusive, a distanciou da Linguística Gerativa (que, apesar das inúmeras diferenças, tem em comum com a LC o foco em questões relativas à relação entre a mente e a linguagem) é justamente a inseparabilidade entre o cognitivo e o social. Sendo assim, a conjunção coordenativa aditiva "e" no título Cognição e Sociedade, juntamente com sua aparente implicação no estabelecimento de uma possível dicotomização entre os dois conceitos, colocou-se como um elemento potencialmente problematizável em si mesmo. No âmbito da Linguística Cognitiva, a cognição é vista como:

inerentemente social e cultural, não apenas em termos de as pessoas compartilharem os tópicos ou os resultados de suas interações, mas em termos das práticas interpretativas por meio das quais as pessoas constituem os tópicos e os resultados de suas interações. ${ }^{1}$ (SAFERSTEIN, 2010, p. 113)

As definições de frame, um conceito central na Linguística Cognitiva, de Lakoff (2004) e de Turner (2001), a seguir, evidenciam o quão imbricadas estão as dimensões socioculturais e cognitivas na formação e reprodução do que Underhill (2012) chama "visão de mundo" (world view), de base inteiramente sociocognitiva:

\begin{abstract}
Frames são estruturas mentais que moldam a maneira com que vemos o mundo. Como resultado, eles moldam os objetivos que traçamos, os planos que fazemos, a maneira que agimos e o que conta como resultados bons ou ruins de nossa ações. Na política, nossos frames moldam nossas políticas sociais e as instituições que formamos para implementar nossas decisões. Mudar nossos frames é mudar tudo isso. Reframing é mudança social. ${ }^{2}$ (LAKOFF, 2004, p. xv)

Operações mentais básicas operam sobre um conjunto cultural e pessoal de conhecimentos. Alguns desses conjuntos serão largamente compartilhados em uma cultura, e certas expressões da língua dessa cultura o evocarão. Cientistas cognitivos chamam esses conjuntos de conhecimentos "frames"3. (TURNER, 2001, p.12)
\end{abstract}

Outros conceitos fundadores da Linguística Cognitiva, como o de MCI (Modelos Cognitivos Idealizados (LAKOFF, 1987)) e até mesmo o de "metáfora conceptual" são, hoje, estudados em sua relação com o ambiente sociocultural e com os construtos ideológicos que evocam. Esses estudos compõem a nova tendência conhecida como "Teoria Crítica da Metáfora", que será discutida mais adiante neste trabalho. Isso não quer dizer que outros tipos de representações cognitivas, mais diretamente relacionadas a experiências sensório-motoras, não sejam alvo de debate sobre a possibilidade de sua

\footnotetext{
${ }^{1}$ [Cognition is inherently social and cultural, not merely in terms of people sharing the topics or the results of their interactions, but in terms of the interpretative practices through which people constitute the topics and results of their interactions].

${ }^{2}$ [Frames are mental structures that shape the way we see the world. As a result, they shape the goals we seek, the plans we make, the way we act and what counts as good or bad outcome of our actions. In politics our frames shape our social policies and the institutions we form to icarry out our policies. To change our frames is to change all this. Reframing is social change.]

${ }^{3}$ [Basic human mental operations operate over cultural and personal assemblies of knowledge. Some of these sets will be widely shared in a culture, and expressions in the culture's language will evoke them. Cognitive scientists call these sets of knowledge "frames".]
} 
universalidade, como as metáforas orientacionais, as metáforas primárias e os esquemas imagéticos. Mesmo assim, em relação à primeira (metáfora orientacional), Lakoff e Johnson (2002 [1980], p. 74) sustentam que, "de um modo geral, as orientações [...] parecem existir em todas as culturas, mas a maneira pela qual os conceitos são orientados, assim como a hierarquia das orientações, variam de cultura para cultura."

Até mesmo a suposta universalidade da metáfora primária, como "INTIMIDADE É PROXIMIDADE”, por exemplo, foi questionada por Martin (2004, p. 202): “Onde está a cultura neste quadro? Todas as culturas têm conceitos para intimidade ou afeição, ou até mesmo atribuem-lhe sentido da mesma maneira? ${ }^{4}$. Segundo a mesma autora, até mesmo o conceito de "corpo", central para a noção de cognição corporificada, não deve pressupor um corpo "a-histórico" e não socioculturalmente situado.

Com base nessas considerações, que emergiram da problematização de uma possível dicotomização conceitual entre "cognição e sociedade", o objetivo deste trabalho foi redirecionado para uma breve reflexão em torno da relação entre os dois conceitos e, principalmente, como essa relação tem sido abordada em alguns estudos da área. Como instrumento teórico-analítico para conduzir essa reflexão, foram acionados conceitos da própria Linguística Cognitiva: MCIs, frames e esquemas imagéticos.

\section{UM OLHAR SOBRE A COGNIÇÃO SOB O VIÉS DA LINGUÍSTICA COGNITIVA}

Um dos pilares da Linguística Cognitiva é o reenquadramento do conceito de "cognição". Tanto no senso comum quanto em estudos mais ortodoxos, fora do âmbito da LC, a cognição é normalmente abordada, em seu sentido dicionarizado (Dicionário Houaiss), como "ato ou efeito de conhecer; processo ou faculdade de adquirir um conhecimento". No Dicionário Informal, a definição é similar: "Ato ou processo de conhecer, inclui estados mentais e processos como pensar, a atenção, o raciocínio, a memória, o juízo, a imaginação, o pensamento, o discurso, a percepção visual e audível, a aprendizagem, a consciência, as emoções."

O que essas definições têm em comum é o fato de a cognição ser vista essencialmente como um processo de aquisição de conhecimento ou de raciocínio, haja vista a menção à memória, à atenção, à imaginação, etc. Sendo assim, a cognição é normalmente associada aos processos envolvidos na aquisição de conhecimento, o que parece pressupor um esquema imagético de "contêiner" para se entender a mente. Nesse prisma, a cognição passaria a ser abordada como os meios de se levar conhecimento a este contêiner. Esse enquadramento pode ser visto como análogo, na perspectiva da cognição e não na da comunicação, à metáfora do canal (REDDY, 1993).

A noção de cognição como processo remete à de desenvolvimento cognitivo, um outro conceito largamente divulgado a partir do paradigma construtivista, cujos dois

\footnotetext{
${ }^{4}$ [Where is culture in this picture? Do all cultures even have concepts of intimacy or affection, let alone give them meaning in then same way? ]

${ }^{5}$ A definição do Dicionário Informal foi aqui incluída porque parece refletir a noção mais aceita no senso comum, além de ser a mais bem avaliada entre as quatro propostas por diferentes usuários. (<http://www.dicionarioinformal.com.br/cogni\%C3\%A7\%C3\%A3o/>. Acesso em 5 maio 2016.
} 
representantes principais, mesmo que vistos como antagônicos em alguns aspectos, seriam Jean Piaget e Vygotsky. Como já é amplamente conhecido, segundo a teoria piagetiana, o desenvolvimento cognitivo seria ancorado na ação e em funções lógicas, seguindo uma gradação de quatro estágios no processo de aquisição de estruturas, que formariam um conjunto unificado de conhecimento ou de habilidades cognitivas. Já em Vygotsky, o desenvolvimento cognitivo é abordado a partir de um viés sociocultural, que atribui um forte papel às interações no ambiente social enquanto propulsoras do desenvolvimento cognitivo. (SANTANA; ROAZZY; DIAS, 2006).

A noção de cognição que fundamenta a Linguística Cognitiva, por sua vez, se afasta da perspectiva, tanto teórica quanto do senso comum, que vincula a cognição à aquisição de conhecimento (inclusive linguístico) e a um conjunto de habilidades cognitivas, muitas vezes associadas à capacidade intelectual. Esse afastamento (e não necessariamente um rompimento radical; afinal, isso acarretaria, nas palavras de Reddy (1993), um "conflito de enquadramento"- frame conflict) se dá por meio da introdução do conceito de "cognição corporificada", e, mais recentemente, a de "cognição situada". Apesar da ênfase atual nesta última, vale lembrar que o conceito de cognição corporificada já contempla um contexto bem mais amplo, incluindo o sociocultural:

\footnotetext{
Ao usar o termo "encarnada/corporificada" queremos destacar dois pontos: primeiro que a cognição depende dos tipos de experiência que decorrem do fato de se ter um corpo com várias capacidades sensório-motoras e, segundo, que estas capacidades sensório-motoras individuais estão inseridas em um contexto biológico, psicológico e cultural bem mais abrangente "7. (VARELA; THOMPSON; ROSCH, 1992, p. 172-173)
}

Conceitos de base da LC, como o de metáfora conceptual, frames, esquemas imagéticos, protótipos, MCIs e até mesmo o de construção gramatical se referem às representações cognitivas (ou modelos mentais mais estáveis, que incluem os chamados "modelos culturais" (van DIJK, 2009) que formam a base de nosso sistema conceptual, ancorando e promovendo a conceptualização da experiência. Como essas representações, por sua vez, emergem das experiências tanto sensório-motoras quanto socioculturais, a mente, nessa perspectiva, não pode ser vista como estando separada do corpo, da língua e do mundo. Nesse sentido, a cognição corporificada é sempre situada em um contexto sociocultural, e esse contexto não se constitui de entidades e/ou situações objetivas, que influenciam diretamente a cognição, pois, segundo van Dijk (2009), essas mesmas entidades e/ou situações são, elas próprias, objetos cognitivos, representações mentais.

\footnotetext{
${ }^{6} \mathrm{O}$ termo em língua inglesa embodied cognition também foi traduzido para o português como cognição encarnada. No entanto, optou-se, neste trabalho, pela expressão cognição corporificada pelo fato de esta ter um uso mais frequente no português brasileiro, como verificado em um site de busca.

${ }^{7}$ [By using the term embodied we mean to highlight two points: first that cognition depends upon the kinds of experience that come from having a body with various sensorimotor capacities, and second, that these individual sensorimotor capacities are themselves embedded in a more encompassing biological, psychological and cultural context.]
} 
3 FRAMES SUBJACENTES À RELAÇÃO ENTRE COGNIÇÃO E SOCIEDADE

Considerando a aparente dicotomia entre os conceitos de sociedade e de cognição, podemos observar que abraçar conceitualmente o entrelaçamento ou até mesmo a inseparabilidade entre os dois conceitos, o que seria coerente com os pressupostos da LC, implicaria, também, um frame conflict, pois a própria maneira pela qual falamos sobre ambos evidencia a quase impossibilidade de se romper conceptualmente (e não conceitualmente, um processo mais consciente) com essa dicotomia. Ou seja, parece haver determinados frames que estruturam sociocognitivamente essa relação e que pressupõem a separação entre os dois conceitos, mesmo que não concordemos, teoricamente, com ela.

O primeiro frame enquadra a cognição como inserida na sociedade, que pode ser pensada como um ambiente, um contexto, uma realidade, um meio, uma situação (expressão licenciada pela metáfora conceptual SITUAÇÃO É LUGAR), uma experiência envolvendo o outro e, finalmente, o mundo.

\section{Figura 1 - Cognição e Sociedade: frame 1 (contêiner) (S= Sociedade C= Cognição)}

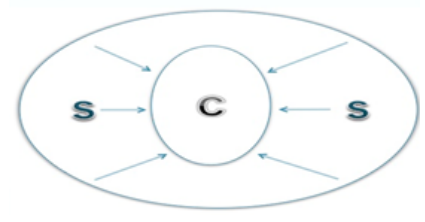

A figura 1 é uma representação esquemática desse enquadre, em que a cognição está inserida em um contêiner, sendo "afetada", "informada", "influenciada", "nutrida", "moldada", "determinada", "constituída", "construída" e até "moldada" por elementos que emergem desse contêiner: "Cognition is always shaped by the social context in which it takes place" (OAKES et al., 2004).

Nessa representação, o elemento "cognição", ao contrário de outras representações que rejeitam, conceitual e epistemologicamente, a influência determinante do contexto sociocultural, colocando a cognição como uma instância mental autônoma, mantém uma relação com este contexto que pode ser também compreendida a partir do esquema imagético de força (TALMY, 2000). Nesta dinâmica de forças, que implica causalidade/mudança, a cognição seria o agonista e a sociedade o antagonista, que exerce força (causa mudança/influência) sobre a primeira.

Um outro frame que parece ser evocado nas representações da relação entre cognição e sociedade é o que emerge do esquema imagético parte-todo (JOHNSON, 1987). Nesse caso, a mente é metonimicamente conceptualizada como o próprio indivíduo, e este pertenceria a um grupo social maior, como família, associação e a própria sociedade. Nesse sentido, a cognição não seria social, e nem manteria uma relação de interdeterminação com o contexto sociocultural: 
Um sistema cognitivo pode ser incorporado em um único indivíduo ou distribuído ao longo de um grande número de indivíduos. Falamos de cognição individual ou de cognição distribuída. Cognição social é um processo cognitivo distribuído sobre todos os membros de uma sociedade, interagindo dentro de uma rede social. A cognição individual também pode ser considerada como cognição distribuída, mas apenas através da rede neural do próprio indivíduo. ${ }^{8}$ (CHAVALARIAS, 2008, p. 54)

\title{
Figura 2 - Frame 2 : Cognição Individual X Cognição Social
}

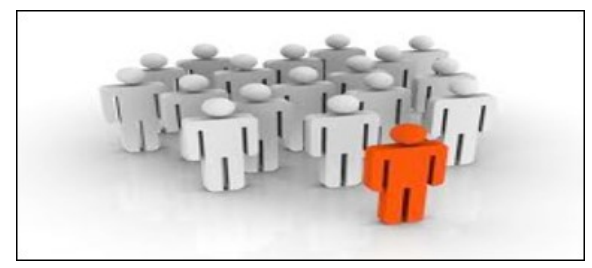

O frame que conceptualiza o indivíduo (e sua mente, numa relação metonímica) como um elemento autônomo, que pertence ao conjunto de elementos que compõem a sociedade, remete à concepção de cognição discutida na seção 1 , ou seja, aquela que aborda este conceito como um processo de aquisição de conhecimento que envolve habilidades cognitivas como atenção, percepção, memória, raciocínio, processamento, entre outras. Assim, a mente do indivíduo é, ao mesmo tempo, a fonte desses processos e o recipiente dos produtos resultantes desses processos: conhecimento, pensamento, ideias e a própria linguagem, sendo este um conhecimento específico de um sistema (ou de um conjunto de regras, ou uma "competência", na perspectiva modular chomskyana), usado para expressar tais pensamentos e ideias.

Essa noção de indivíduo, inerente ao conceito de "cognição individual", em contrapartida à de cognição compartilhada ou distribuída, é hoje contestada no âmbito de várias áreas das ciências humanas e sociais. Segundo Ehrenberg (2009),

\begin{abstract}
Essas filosofias ou essas sociologias subjacentes às ciências neurais permanecem prisioneiras da oposição entre o indivíduo e a sociedade ou entre um interior subjetivo e um exterior objetivo. Ora, o conceito de pessoa não separa o indivíduo e a sociedade, como também não separa um interior subjetivo e um exterior objetivo, ele remete indiretamente a um individuo empírico; ele designa e descreve a possibilidade de ocupar as três posições pessoais da pessoa verbal: para poder dizer 'eu falo', é necessário ser capaz de se reconhecer, segundo o caso, como aquele que fala (eu), aquele a quem se fala (tu) e aquele de qual se fala (ele), a nãopessoa ou o mundo que forma "o membro faltante da correlação de pessoa". O conceito de pessoa é um conceito puramente relacional (EHERENBER, 2009, p. 124 ).
\end{abstract}

Mesmo no âmbito das ciências neurais, a que Eherenberg se refere na citação acima, as dicotomias indivíduo $\mathrm{x}$ grupo social, interior $\mathrm{x}$ exterior e, portanto, cognição $\mathrm{x}$ sociedade, que é a que nos interessa mais particularmente neste trabalho, estão sendo

8[A cognitive system can be embedded in a single individual or distributed over a large number of individuals. We speak of individual cognition or distributed cognition. Social cognition is a cognitive process distributed over all members of a society, interacting within a social network. Individual cognition as well might be considered as distributed cognition over a neural network]. Para uma discussão mais aprofundada sobre cognição distribuída, ver Salomon (1997). 
empiricamente desafiadas com a descoberta dos chamados "neurônios-espelhos" que, segundo Salomão (2007), estão sendo abordados como a base material das experiências da intersubjetividade e da empatia. Um neurônio espelho é aquele que dispara quando um animal age e, também, quando o animal apenas observa um outro se engajar nesta mesma ação (KEYSERS, 2011).

Esta descoberta parece corroborar a hipótese da cognição corporificada, pilar da Linguística Cognitiva, em sua base experiencialista, pois, de algum modo, comprova que algo tão individual quanto um neurônio, em um cérebro específico, dispara pela simples imaginação, através do contato intersubjetivo. Ou seja, até mesmo no âmbito das neurociências, a mente não está sendo mais abordada em uma perspectiva individual, pois a inserção e a interação sociais afetam, de algum modo, o próprio funcionamento das redes neurais. Sendo assim, do ponto de vista da cognição, o indivíduo caracterizado na figura 2 não seria apenas um elemento pertencente a um conjunto de outros indivíduos com quem convive mais ou menos de perto; mas sim um elemento que se constitui, subjetiva e socialmente, pela relação que mantém com os outros indivíduos, com as trocas simbólicas que com eles estabelece e pelas experiências sensório-motoras, que, sempre mediadas pelo simbólico (logo, pelo outro), servem de matéria-prima para conceptualizações (“distribuídas”) de suas experiências.

\section{ARTICULAÇÃO ENTRE COGNIÇÃO E SOCIEDADE: O CASO DA METÁFORA EM USO}

Retomando um dos pontos centrais discutidos até o momento, ou seja, a dicotomização entre cognição e sociedade, reafirmamos que esta não encontra suporte nos postulados da Linguística Cognitiva, que tem a cognição corporificada como um de seus principais fundamentos. Isso não impede, no entanto, que essa dicotomização se "infiltre" em várias proposições encontradas nessa área do conhecimento, uma vez que os frames que sustentam essa separação estão imbricados em nosso pensamento e inscritos em nossa linguagem. Frame conflicts não podem ser radicalmente promovidos apenas por mudanças de paradigmas, por mais teoricamente consistentes que esses possam ser.

Mesmo assim, as pesquisas desenvolvidas nas diversas ilhas da LC (GEERAERTS, 2006) apontam para a permanente articulação entre a cognição e a sociedade, e tanto um conceito quanto o outro são abordados sob ângulos diversos, de acordo com o objeto e as perguntas que norteiam a pesquisa.

O quadro 1 apresenta uma explicitação, bastante esquemática, e, portanto, inevitavelmente reducionista, mas que se propõe a iluminar essas diferentes perspectivas, no caso específico do estudo da metáfora - um dos principais objetos de estudo da LC para que pesquisas na área possam ser compreendidas e cotejadas a partir de suas possíveis similaridades e diferenças. $\mathrm{O}$ quadro parte da distinção entre sistema e uso, proposta por Steen (2006). 
Quadro 1 - Níveis de cognição: sistema x uso

\begin{tabular}{|l|l|}
\hline Nível Estável (Sistema) & Nivel Episódico (Uso) \\
\hline Metaforas Conceptuais & Metáforas Situadas \\
\hline Frames off-line, MCIs, Esquemas Imagéticos & Frames online \\
\hline Discurso & discurso \\
\hline
\end{tabular}

O primeiro nível, chamado, no quadro 1, nível estável da cognição, refere-se ao que Lakoff (1987) chama, de maneira abrangente, "sistema conceptual". Nesse nível de cognição estariam as representações mentais mais estáveis, como as elencadas por Lakoff (1987): os MCIs, os protótipos, os esquemas imagéticos, as metáforas conceptuais e os frames. Neste quadro, proponho a especificação de frame no nível estável como frame off-line, ou seja, uma representação socialmente compartilhada e essencialmente inconsciente, como seria o caso das outras representações do mesmo grupo. Ainda nesse nível, teríamos o Discurso, que seria a inscrição das representações estáveis na linguagem, enquanto sistema.

Já o nível episódico se refere ao evento discursivo em si, ou seja, à cognição e à linguagem em uso. As metáforas, nesse nível, por exemplo, ao contrário das conceptuais, seriam situadas, de natureza mais deliberada e menos convencional, fazendo parte da tessitura textual e da construção de frames online ou, para usar um termo da Linguística Textual, da "construção do objeto de discurso" (MONDADA, 2002). Finalmente, há o discurso (que, no quadro 1, teria o Discurso como contraparte, no nível estável) e seria o próprio evento discursivo, em pleno acontecimento. Nesse nível, as instâncias do nível estável seriam evocadas e articuladas àquelas presentes na cognição online. De fato, não há um nível sem o outro: a separação é meramente teórica e analiticamente operacional.

Há de se evitar, em uma análise que tenha como foco a linguagem metafórica, fazer afirmações sobre uma metáfora situada como se esta fosse conceptual, ou vice-versa, pelo fato de ambas estarem inseridas em níveis de cognição distintos, mesmo que relacionados: um episódico e outro estável, respectivamente. Essa distinção é relevante, pois implica questões sobre a convencionalidade, a disseminação sociocognitiva e discursiva e o grau de deliberalidade e/ou consciência no uso da metáfora.

No entanto, as possíveis articulações que entrelaçam a metáfora conceptual e a metáfora situada podem ser, em um estudo que tenha esse propósito, identificadas e analisadas. É bastante comum nos deparamos com um "nicho metafórico" (VEREZA, 2013b), um conjunto de enunciados construídos e articulados, a partir de mapeamentos textuais online, em que metáforas situadas são licenciadas por metáforas conceptuais subjacentes. Segue um exemplo de nicho metafórico, um fragmento retirado de um texto publicado em um blog bastante popular', o Bolsa de Mulher:

${ }^{9} \mathrm{O}$ blog recebe, mensalmente, a visita de 41 milhões de usuários, segundo o Google Analytics. 


\section{SEM RUMO....}

(por Administradores)

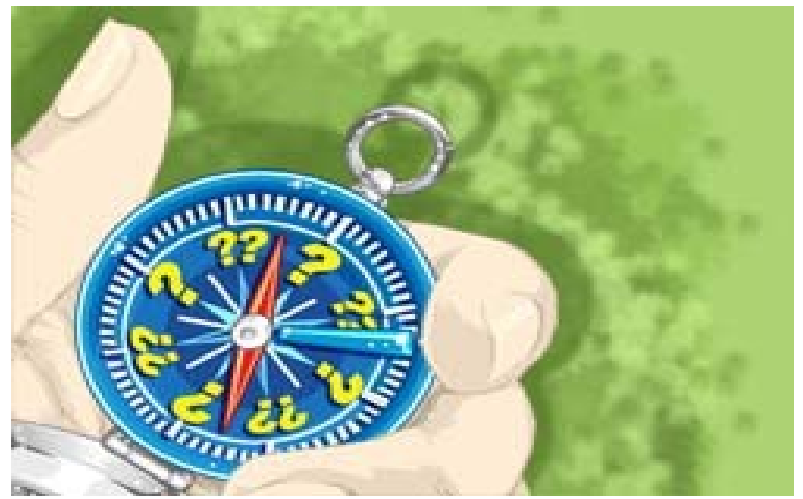

[...] Pode parecer estranho, mas se sentir perdido no mundo, completamente sem rumo, sem saber o que fazer e o que querer é uma experiência pela qual muita gente já passou - ou ainda passa [...] Em primeiro lugar, para que uma pessoa se ache, antes é preciso que ela tenha estado perdida. Ninguém passa a vida inteira com os pés firmes no chão, satisfeito com o que está fazendo e em harmonia com o mundo e com seus sentimentos. É mais que normal as pessoas passarem por fases em que parecem estar desencontradas, sem chão. Quem estiver nessa situação deve lembrar que não é o único a passar por isso e que reencontrar o caminho de volta é somente uma questão de tempo. [...] Só que, pelo visto, não é assim com todo mundo, não. Tem gente que parece não sair nunca do período de desorientação. Uma coisa é estar sem rumo, outra é ver os anos passarem, passarem e nada de descobrir o caminho certo. Fonte: <http://www.bolsademulher.com/estilo/sem-rumo>. Acesso em: 25 maio 2016.

A argumentação tecida no texto, de claro teor de autoajuda, é desenvolvida a partir de mapeamentos locais, textualmente explorados, com base em uma metáfora situada (plano episódico) que conceptualiza, localmente, a "instabilidade na vida" (profissional, emocional, etc.) como "desorientação no espaço". Essa metáfora, apesar de situada, é bastante convencional, e está ancorada em metáforas conceptuais (plano estável): A VIDA É UMA VIAGEM/CAMINHO (o caminho de volta; o caminho certo); OBJETIVO NA VIDA É RUMO NO CAMINHO (sem rumo; perdido no mundo, desorientação); ESTABILIDADE EMOCIONAL É ESTABILIDADE FÍSICA (com os pés firmes no chão; sem chão). A ilustração, que mostra uma pessoa (metonimicamente representada pela mão) segurando uma bússola, seria, também, uma instanciação, explorada imageticamente, dessas metáforas, principalmente das duas primeiras.

Outros elementos de natureza mais estável são acionados, como o frame que estrutura os domínios fontes "viagem" e "caminho", mais concretos, além de frames (ou MCIs) de natureza sociocultural, como "objetivos de vida" e "inserção social". Estes últimos, ao se articularem com os de nível mais episódico, geram tanto expectativas quanto as consequentes noções do que seriam felicidade e frustração, com efeitos psicológicos, como argumentado no próprio texto.

Este nicho metafórico, portanto, parece evidenciar a articulação, no evento discursivo, entre os dois níveis de cognição aqui propostos, um mais estável, mais 
difundido socioculturalmente e mais inconsciente; e o outro mais episódico, local e fruto de um ato comunicativo mais deliberado.

No que concerne à relação entre cognição e sociedade, foco deste artigo, esta também pode ser abordada, no contexto do exemplo analisado, a partir dos dois níveis propostos. Em primeiro lugar, a cognição local é estabelecida como instrumento para se tecer a "argumentatividade" (GUIMARÃES, 2013) pretendida, ou seja, explorar discursiva e retoricamente um dado ponto de vista, por meio de mapeamentos locais, marcados linguisticamente pela escolha, aparentemente deliberada, de determinados veículos metafóricos. Em segundo lugar, a relação entre a cognição e a sociedade é evidenciada pela evocação, não necessariamente consciente, de instâncias do nosso sistema conceptual, como no caso de frames e de metáforas conceptuais, sejam essas de base sensório-motora, sejam de base sociocultural. É nesse último aspecto que a cognição é fundamentalmente ideológica, revelando a sua relação interconstitutiva com a sociedade. Essa relação é ressaltada por Lakoff, que acredita que

toda e qualquer ideologia é um sistema conceptual de algum tipo, inclusive um sistema moral. No entanto, ideologias possuem aspectos tanto conscientes quanto inconscientes. Se perguntássemos para uma pessoa, com uma dada ideologia política, no que ela acredita, ela vai nos dar uma lista de crenças e, talvez, algumas generalizações. Um linguista cognitivo, ao ouvir a sua resposta, provavelmente atentará para os frames e metáforas inconscientes por trás das suas crenças conscientes. Ao meu ver, essa é a parte interessante das ideologias: a parte inconsciente. É a respeito dela que o linguista cognitivo pode trazer alguma contribuição. ${ }^{10}$ (LAKOFF apud OLIVEIRA, 2001, p. 37).

\section{CONSIDERAÇÕES FINAIS}

Concluo este trabalho ousando discordar da proposição expressa no último período da citação acima, apesar de ter usado essa citação por compartilhar a visão de Lakoff no que diz respeito à relação de interdeterminacão entre sistema conceptual e ideologia. $\mathrm{O}$ enunciado específico, a que me refiro, parece remeter à aparente rejeição, por parte de setores mais ortodoxos da LC, do enfoque analítico nos aspectos ditos da enunciação (no uso e não no sistema; no discurso e não no Discurso, seguindo o esquema aqui proposto), como não sendo de interesse para linguistas cognitivos. Essa rejeição parece ser fruto do deslocamento do lócus da metáfora da linguagem para o pensamento que a visão cognitivista promoveu. Para tanto, foi necessário romper com a visão tradicional de metáfora enquanto figura meramente estilística, poética ou retórica, sem qualquer função cognitiva. A partir dessa ruptura inicial, provavelmente necessária para a quebra de paradigma que a Teoria da Metáfora Conceptual introduziu, o estudo das metáforas e

\footnotetext{
${ }^{10}$ [Any ideology is a conceptual system of a particular kind, including a moral system. However, ideologies have both conscious and unconscious aspects. If you ask someone with a political ideology what she believes, she will give a list of beliefs and perhaps some generalizations. A cognitive linguist, looking at what she says, will most likely pick out unconscious frames and metaphors lying behind her conscious beliefs. To me, that is the interesting part of ideologies: the unconscious part. It is there that cognitive linguists have a contribution to make].
} 
outras figuras no funcionamento discursivo ficou em segundo plano. As representações mentais, como os frames, MCIs e metáforas conceptuais passaram a ser consideradas como estruturas fundantes da construção de sentido, principalmente no que se refere à categorização. E é nesse âmbito conceptual que, segundo o que podemos depreender da citação de Lakoff, a relação da cognição com a sociedade interessaria à Linguística Cognitiva.

No entanto, creio que os recentes caminhos traçados pela Linguística Cognitiva, principalmente nos estudos cognitivo-discursivos da metáfora (CAMERON; MASLEN, 2010; MULLER, 2008; CHARTERIZ-BLACK, 2004; SEMINO, 2008; VEREZA; MOURA; ESPÍNDOLA, 2013, entre outros), que desvelam a complexidade do entrelace entre o sistema conceptual e a construção online de objetos de discurso no evento discursivo, apontam para a necessidade de se buscar compreender as possíveis formas de articulação entre o mais ou menos estável, e o mais ou menos consciente. Ressalto aqui o uso do modalizador "mais ou menos", uma vez que não há, de fato, estabilidade ou instabilidade plena, mas sim um continuum entre os dois níveis. Como já aqui mencionado, a proposta de uma separação entre eles é feita por razões teórica e metodologicamente operacionais, e pelo fato de acreditarmos que instrumentos conceptuais têm o potencial de alavancar novos entendimentos, desde que reconheçamos suas limitações. E é dentro dessa perspectiva que emerge, neste trabalho, da problematização da dicotomização entre sociedade e cognição, que creio ser possível conduzir investigações que visem a compreender alguns aspectos da articulação entre as duas instâncias, no discurso. Nesse processo, deparamo-nos, inevitavelmente, com a grande complexidade envolvida nessa articulação, o que nos impulsiona a buscar novas perguntas e novos instrumentos (ou reformulações destes) para tentar explorá-la.

\section{REFERÊNCIAS}

CAMERON, L.; MASLEN, R. (Org.). Metaphor analysis: research practice in applied linguistics, social sciences and the humanities. Londres: Equinox, 2010.

CHARTERIS-BLACK, J. Corpus approaches to critical metaphor analysis. Nova York: Palgrave, 2004.

CHAVALARIAS, D. From individual cognition to social cognition. In: BOURGINE, P.; CHAVALARIAS, D.; PERRIER, E. French road for complex systems. Paris: Institut des Systèmes Complexes, 2008. p. 54-55. Disponível em: $<$ https://arxiv.org/ftp/arxiv/papers/0907/0907.2221.pdf $>$ Acesso em: 26 out. 2016.

EHREMBERG, A. O cérebro social: quimera epistemológica e verdade sociológica. Periferia, v. 1, n. 2 , p.114-131, 2009.

FILLMORE, C. Frame semantics. In: GEERAERTS, D. Cognitive Linguistics: basic readings. Berlim: Mouton de Gruyter, 2006. p. 373-400.

GEERAERTS, D. Cognitive Linguistics: basic readings. Berlim: Mouton de Gruyter, 2006.

GUIMARÃES, E. Argumentatividade e argumentação. Desenredo, v. 9, n. 2, p. 271-283, 2013.

JOHNSON, M. The body in the mind: the bodily basis of meaning, imagination and reason. Chicago: Chicago University Press, 1987.

KEYSERS, C. The Empathic brain. Amsterdam: Social Brain Press, 2011.

KÖVECSES, Z. Metaphor and culture. Cambridge: Cambridge University Press, 2005. 
LAKOFF, G. Don't think of an elephant. White River Junction: Chelsea Green Publishing, 2004.

Women, fire, and dangerous things: what categories reveal about the mind. Chicago: The University of Chicago Press, 1987.

LAKOFF, G.; JOHNSON, M. Metaphors we live by. Chicago: The University of Chicago Press, 1980.

Metáforas da vida cotidiana. Tradução do Grupo de Estudos da Indeterminação e da Metáfora. Campinas/São Paulo: Mercado de Letras/Editora da PUC-SP, 2002.

MARTIN, E. Talking back to neuro-reductionism. In: THOMAS, H.; JAMILAH, A. Cultural Bodies: ethnography and theory. Oxford: Blackwell, 2004. p.190-212.

MONDADA, L. Construction des objets de discours et catégorisation: une approche des processus de référenciation. Tradução de Mônica Magalhães Cavalcante. Revista de Letras, v. 1/2, n. 24, p. 118-130, 2002.

MÜLLER, C. Metaphors dead and alive, sleeping and waking: a dynamic view. Chicago: University of Chicago Press, 2008.

OLIVEIRA, R. P. Language and ideology: interview with George Lakoff. In: DIRVEN, R.; HAWKINS, B.; SANDIKCIOUGLU, E. Language and ideology. Vol. 1. Amsterdam: John Benjamins, 2001. p. 23-47. REDDY, M. J. The conduit metaphor: a case of frame conflict in our language about language. In: ORTONY, A. (Org.). Metaphor and thought. 2. ed. Cambridge: Cambridge University Press, 1993. SAFERSTEIN, B. Cognitive Sociology. In: JASPERS, J.; OSTMAN, J.-O.; VERSCHUEREN, J. (Org.). Society and language use. Amsterdam: John Benjamins, 2010. p. 113-126.

SALOMÃO, M. M. M. Lanterna na proa: sobre a tradição recente nos estudos da linguística. Gragoatá, v. 23, p. 27-52, 2007.

SALOMON, G. Distributed cognitions: psychological and educational considerations. Cambridge: Cambridge University Press, 1997.

SANTANA, S. M.; ROAZZI, A.; DIAS, M. das G. B. B. Paradigmas do desenvolvimento cognitivo: uma breve retrospectiva. Estudos de Psicologia, v. 11, n. 1, 2006.

SEMINO, E. Metaphor in discourse. Cambridge: Cambridge University Press, 2008.

STEEN, G. Metaphor in applied linguistics: four cognitive approaches. D.E.L.T.A., v. 22, n. especial, p. 21-44, 2006.

What does 'really deliberate' really mean? More thoughts on metaphor and consciousness. Metaphor and the Social World, v.1, n. 1, p. 53-56, 2011.

TALMY, L. Toward a Cognitive Semantics. Cambridge: The MIT Press, 2000.

TURNER, M. Cognitive dimensions of social sciences. Oxford: Oxford University Press, 2001.

UNDERHILL, J. Creating worldviews: metaphor, ideology and language. Edimburgo: Edinburgh University Press, 2013.

VAN DIJK, T. A. Society and Discourse: how social contexts influence text and talk. Cambridge: Cambridge University Press, 2009.

VARELA, F.; THOMPSON, E.; ROSCH, E. The embodied mind: Cognitive Science and human experience. Cambridge: The MIT Press, 1992.

VEREZA, S. Cry me a river: metaphoric hyperboles in the interface between discourse and cognition. Gragoatá, v. 40, p.175-196, 2016.

. Entrelaçando frames: a construção do sentido metafórico na linguagem em uso. Cadernos de Estudos Linguísticos, v. 55, p.109-124, 2013a.

Discourse, cognition and figurative language: Exploring metaphors in political editorials In: Comunicação Política e Econômica: dimensões cognitivas e discursivas. Publicações da Faculdade de Filosofia Universidade Católica Portuguesa, v.1, p. 383-394, 2013b.

VEREZA, S.; MOURA, H. M. M.; ESPÍNDOLA, L. C. Metáfora e contexto: entre o instável e o estável. Interdisciplinar, v. 17, p. 1-14, 2013. 
Recebido em: 04/07/16. Aprovado em: 29/07/16.

Title: Cognition and society: a view from the perspective of Cognitive Linguistics

Author: Solange Coelho Vereza

Abstract: This work proposes a reflection on the relationship between cognition and society, from the perspective of Cognitive Linguistics (CL). The concept of embodied cognition, one of the pillars of cognitive linguistics, challenges any possible dichotomy between the two concepts, since cognitive representations, such as frames, idealized cognitive models (ICMs) and conceptual metaphors would be anchored in conceptualizations of both sensory-motor and sociocultural experiences. The search for the understanding of the relationship between society and cognition, however, must, according to Gibbs (2001), must leave the exclusive realm of thought and find the world. For this reason, we propose an approach in line with recent cognitive-discursive trends, which seeks theoretical and methodological tools to systematize the investigation of such articulation. Among these instruments, the distinction between system and use, episodic and stable cognition, online and offline frames and situated and conceptual metaphors has proved to be both theoretically and analytically productive.

Keywords: Sociocognition. Metaphor. Discourse.

Título: Cognición y sociedad: una mirada bajo el punto de vista de la Lingüística Cognitiva Autor: Solange Coelho Vereza

Resumen: Este trabajo propone una reflexión sobre la relación entre cognición y sociedad, bajo la perspectiva de la Lingüística Cognitiva (LC). El concepto de cognición encarnada (embodied cognition), uno de los pilares de la LC, pone en jeque cualquer posibilidad de dicotomización entre los dos conceptos, una vez que representaciones cognitivas como frames, modelos cognitivos idealizados (MCI) y metáforas conceptuales serían ancladas em conceptualizaciones de experiencias, tanto sensorio-motoras, cuanto socioculturales. La búsqueda de la comprensión de la articulación entre el social y el cognitivo, sin embargo, debe, de acuerdo con Gibbs (2001), salir del ámbito exclusivo del pensamento y encontrar el mundo. Por esta razón, propomos un abordaje alineada con el reciente tendencia cognitivodiscursiva, que busca instrumentos teóricos y metodológicos para sistematizar el estudio de esa articulación. Entre ellos, la distinción entre sistema y uso, cognición episódica y estable, frames em línea y fuera de línea, y metáforas situadas y conceptuales tiene se muestrado teórica y analíticamente productiva.

Palabras-clave: Sociocognición. Metáfora. Discurso.

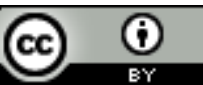

Este texto está licenciado com uma Licença Creative Commons Atribuição 4.0 Internacional. 